\title{
Experiencia en la preparación de investigadores como autores y revisores de artículos científicos
}

\author{
Esperanza Asencio Cabot \\ Nilda Ibarra López \\ Universidad Central "Marta Abreu" de Las Villas, Cuba
}

CASE REPORT

\begin{abstract}
Resumen
El artículo aborda la experiencia desarrollada en la Universidad Central "Marta Abreu" de Las Villas, Cuba, con el objetivo de diseñar e implementar un entrenamiento de posgrado para contribuir a la elevación de la preparación de investigadores experimentados y líderes científicos en el área de las Ciencias Pedagógicas en la elaboración y revisión de artículos en revistas educativas de impacto internacional. Se insiste en la importancia actual del tema tratado y se analiza el diseño metodológico de la experiencia, así como la caracterización del programa y la implementación de actividades docentes. Se presentan los resultados obtenidos y se destaca la medición de los indicadores a partir del desempeño de las personas entrenadas en el rol de autor y en el rol de revisor, lo que permitió el análisis por personas entrenadas y por indicadores, considerando también los datos cualitativos recogidos durante el proceso. En general, se demostraron discretos avances en los personas entrenadas con relación a la elaboración y revisión de artículos, ya que aún se manifiestan limitaciones; sin embargo, se reconoce la influencia que tuvo el entrenamiento en la toma de conciencia de los participantes acerca de la necesidad de elevar el rigor y la calidad en las producciones derivadas de los resultados de las investigaciones para alcanzar la publicación de sus trabajos en revistas reconocidas por la comunidad científica.
\end{abstract}

Palabras clave

Artículos científicos; entrenamiento de posgrado; preparación de investigadores; revistas científicas; revistas educativas; rol de autor; rol de revisor

\section{Experience in training researchers as authors and reviewers of research papers}

\begin{abstract}
The paper presents the experience developed in the Central University "Marta Abreu" of the Villas, Cuba, with the objective of to design and to implement a postgraduate training in order to elevate the preparation of experienced researchers and scientific leaders in the area of Pedagogical Sciences in both elaboration and revision of papers in educative journals. The importance of the treated subject in nowadays is point out, as well as the methodological design of the experience, the characterization of the program and the implementation of educational activities. There are presented the obtained results and is pointed out the performance of the participants in the role of both author and reviewer, which allowed the analysis by both trainers and indicators, considering also the gathered qualitative data during the process. In general, discreet advanc es were demonstrated in the participants, since still limitations are pronounced; however, it is recognized the influence that had the training in the taking of conscience of the participants about the necessity to elevate the rigor and the quality in the productions derived from the results of the research to reach the publication of its works in journals recognized by the scientific community.
\end{abstract}

Keywords

Educative journals; postgraduate training; researcher's preparation; research papers; role of author; role of reviewer; scientific journals

\section{Introducción}

La publicación de resultados científicos constituye una parte esencial del proceso de investigación; al respecto, se refiere el destacado investigador R. Day cuando afirma: 


\begin{abstract}
Un experimento científico, por espectaculares que sean sus resultados, no termina hasta que sus resultados no se publican. De hecho, la piedra angular de la filosofía de la ciencia se basa en la premisa fundamental de que las investigaciones originales tienen que publicarse; solo así pueden verificarse los nuevos conocimientos científicos y añadirse luego a la base de datos que llamamos precisamente conocimientos científicos. (Day, 2005: 9)
\end{abstract}

Las revistas científicas constituyen uno de los principales canales para la comunicación y difusión de los resultados de investigación, así como en la institucionalización social de la ciencia en la mayoría de los campos del conocimiento y su prestigio depende de las bases de datos en las que hayan logrado su indexación, atendiendo a los criterios de calidad exigidos y su impacto en la comunidad científica (Miguel, 2011; Córdoba, 2005; Villamón, et al., 2005).

Las universidades modernas que hoy exhiben más prestigio en el mundo son aquellas que, además de su función en la formación de profesionales, ofrecen altos productos científicos evidenciados por la calidad y visibilidad de sus publicaciones (Sánchez, 2006). Las publicaciones en revistas científicas constituyen uno de los principales índices del desarrollo científico de una región, país, institución o del científico mismo (Valera, 2005; Gómez, Jiménez \& Moreles, 2014). Justamente, los procesos de evaluación y de acreditación de las universidades en el mundo contemporáneo, enfatizan entre los aspectos a medir, los relacionados con la investigación y las publicaciones en revistas científicas indexadas en bases de datos de impacto internacional (Gil, Morales \& Basantes, 2015; Aramburuzabala, Reyes \& Ángel, 2013; Suárez, Escalona \& Boza, 2012).

Lo expresado con anterioridad, justifica la necesidad de que los investigadores desarrollen destrezas y habilidades en la escritura científica. Sin embargo, no siempre se le brinda la atención que requiere la publicación de artículos en el proceso de la investigación. Enseñar a hacer investigación no es una tarea fácil; por tanto, es común que los procesos de formación escolarizados solo se dediquen a mostrar cómo producir conocimiento dejando fuera de sus agendas las tareas relacionadas con la publicación (Gómez, Jiménez \& Moreles, 2014).

Uno de los grandes problemas que enfrentan los investigadores cuando tienen que dar a conocer sus resultados, está precisamente en la preparación de los artículos para enviar a revistas, pues no han desarrollado suficientemente las habilidades necesarias para la producción de textos científicos y no siempre tienen la preparación suficiente para la búsqueda y selección de las revistas donde enviar sus trabajos (Cervantes \& Martínez, 2014) y menos aún para desempeñarse como revisores (Sabaj, 2009). Por lo general, los investigadores no han recibido ninguna asignatura dirigida hacia la redacción y la comunicación científica durante su formación académica y no han asistido a ningún curso de posgrado dirigido a esos fines (Antúnez et al., 2012; Alfonso, 2004; Cordero, et al., 2009; Santovenia \& Linares, 2011; Gutiérrez et al., 2009).

La preparación de un manuscrito, desde su fase inicial hasta su publicación final, suele ser un proceso arduo; la habilidad en la composición escrita, es de hecho, bastante compleja y más aún en el caso del texto científico, que exige presentar un discurso coherente, concreto, y ordenado, utilizando los términos adecuados, para poder trasmitir a los lectores las ideas de los autores (Espino, 2015; Alfonso, 2004; Hernández, 2010; Zapata \& Velásquez, 2008). Este proceso se complejiza más en el contexto actual, donde el dominio de Internet y sus servicios, ha determinado que la mayoría de las revistas de impacto internacional circulen en la web, lo que exige del dominio de la redacción para leer en línea, así como de contenidos actualizados en la gestión de la información (Ortega, Hernández, y Tobón, 2015; Villagrán, 2009; Antúnez, 2006; Cordero, et al., 2009).

En términos generales, la productividad global de las producciones científicas de los profesores-investigadores a nivel internacional no es muy abundante (Cordero, et al., 2009), de ahí que el índice de artículos constituya una de las dimensiones más difíciles de lograr, para una buena parte de ellos (Cordero, et al., 2009; Mainegra \& Miranda, 2012). Con vista a solucionar estas dificultades, muchas universidades del mundo han organizado diversas variantes de superación profesional, tales como: diplomados, cursos presenciales y online, seminarios, talleres, estrategias, sistema de acciones, cursos-talleres, entre otras (Alfonso, 2004; Antúnez, 2006; Antúnez, et al., 2012; Cordero, et al., 2009; Estrada, 2006; Espino, 2015; Fonseca, Lías, \& Gamboa, 2015; Gutiérrez, et al., 2009; Hernández, 2010; Mainegra \& Miranda, 2012). 
En particular, en el contexto de las facultades pedagógicas de la Universidad Central "Marta Abreu" de Las Villas, los índices de publicaciones de los profesores-investigadores en revistas educativas de impacto internacional no escapaban a las limitaciones mencionadas, por lo cual se hizo necesario emprender un programa de acciones dirigidas a elevar la preparación de los docentes para promover publicaciones en dichas revistas. En general, en el programa fueron concebidas diversas acciones, tanto de orden metodológico como de superación profesional, las que estaban en correspondencia con el nivel de partida de los participantes; precisamente el presente trabajo se enmarca en la temática referida.

Es importante declarar los límites de este artículo, el cual, aunque aborda acciones de superación, se enfoca esencialmente hacia el objetivo de diseñar e implementar un entrenamiento de posgrado para contribuir a la elevación de la preparación de investigadores en el área de las Ciencias Pedagógicas en la elaboración y revisión de artículos en revistas educativas de impacto internacional.

\section{Metodología que sustenta la experiencia}

En este epígrafe se analizará la metodología que sustenta la experiencia en la concepción e implementación del entrenamiento de posgrado. Para ello se describirá brevemente el proceso de determinación de necesidades, que permitió precisar el problema y el objetivo, así como los demás elementos del diseño metodológico.

\subsection{El proceso de determinación de necesidades}

Al referirse al proceso de formación de investigadores en el área de la educación, Varela (2005) reconoce que la atención al componente informacional es muy deficiente, y que, a pesar de que en los últimos años se ha producido una gran cantidad de textos de Metodología de la Investigación Educativa (MIE), ninguno hace énfasis en lo referido a la publicación de los resultados. Justamente, el proceso de determinación de necesidades que se presenta a continuación demostró esa aseveración en el contexto declarado con anterioridad.

En la etapa inicial del proceso se profundizó en el estudio de los documentos relacionados con el trabajo científico de los profesores de las facultades pedagógicas; este análisis evidenció la baja circulación internacional de las publicaciones de los docentes y las limitaciones en el número de publicaciones en revistas nacionales. Por otro lado, se aplicaron diversos métodos empíricos tales como: observaciones, entrevistas a docentes, entrevistas grupales a colectivos departamentales, encuestas, entre otros, que permitieron identificar las deficiencias que presentaban los docentes; entre las dificultades se destacaron: el nivel de actualización y diversificación de las fuentes de información utilizadas, el asentamiento de citas y referencias, la redacción y uso de las reglas ortográficas, así como la estructura del artículo.

El estudio, incluyó además un grupo de acciones de investigación con el propósito de obtener criterios acerca de la contribución de la MIE y la tutoría del trabajo científico a la preparación de los docentes-investigadores para lograr publicaciones en revistas; este trabajo fue realizado en la Maestría en Ciencias Pedagógicas y en el Doctorado Curricular Colaborativo en Ciencias Pedagógicas. El análisis de los datos obtenidos demostró carencias en el tratamiento y actualización de la información científica en las disciplinas de MIE y en la tutoría del trabajo científico, lo que demostró que estas vías no siempre ofrecen la contribución necesaria en la preparación de los investigadores para enfrentar el proceso de publicación de artículos en revistas científicas.

Asimismo, constituyó un referente importante en la determinación de necesidades, el estudio bibliométrico realizado por el Centro de Documentación e Información Pedagógica de la propia sede universitaria (Asencio \& Ibarra, 2013) para conocer el empleo de las publicaciones en revistas de impacto internacional como fuentes bibliográficas en las tesis doctorales. En general, los resultados de este estudio demostraron que el empleo de estas revistas en las tesis doctorales fue significativamente pobre y además se apreciaron dificultades en los asentamientos bibliográficos en lo que respecta al completamiento de los datos requeridos, así como errores en las direcciones electrónicas en las revistas incluidas en la bibliografía, entre otros aspectos.

Derivado del estudio realizado, quedó evidenciada la situación problemática que se presentaba en el contexto de las facultades pedagógicas en relación con la preparación de los docentes-investigadores para lograr la publicación de artículos. Estas limitaciones señaladas afectaban no solo a los investigadores noveles, sino también a los líderes científicos en el campo de las Ciencias Pedagógicas, que como investigadores más experimentados, se desempeñaban como directivos en proyectos de investigación y como tutores en maestrías 
y doctorados, así como en otras tareas relacionadas con el trabajo científico; por estas razones la experiencia fue dirigida, en particular hacia dichos investigadores.

\subsection{Diseño metodológico de la experiência}

El proceso de determinación de necesidades descrito permitió precisar el problema científico de la experiencia, el que se define a continuación:

¿Cómo contribuir a la preparación de los líderes científicos e investigadores experimentados en el área de las Ciencias Pedagógicas para lograr la publicación de artículos en revistas educativas de impacto internacional?

Derivado del problema fue declarado como objetivo:

Diseñar e implementar una propuesta de entrenamiento de posgrado, para elevar la preparación de los líderes científicos e investigadores experimentados en el área de las Ciencias Pedagógicas para lograr la publicación de artículos en revistas educativas de impacto internacional.

Con relación, al diseño metodológico de la experiencia, este se acerca al enfoque de la investigación-acción, ya que constituyó un proceso construido desde y para la práctica, para incidir en la formación de los investigadores posibilitando su mejora continua y la vinculación entre la teoría y la práctica (Cruz, 2015). La investigaciónacción en general se considera como una espiral de pasos, entre los que se destacan: la planificación, la implementación y la evaluación, que tiene amplias posibilidades como herramienta metodológica para estudiar la realidad educativa, mejorar su comprensión y al mismo tiempo lograr su transformación (Colmenares \& Piñero, 2008).

Es preciso aclarar que el diseño experimental utilizado el trabajo que se presenta, fue de un pre-experimento con una sola medición realizada después de aplicar el estímulo, lo cual implicó un nivel mínimo de control (Sampiere, Collado \& Lucio, 2006); por esa razón, se ubica en la categoría de experiencia de intervención en la práctica, aunque se aplican métodos y procedimientos de investigación desde una perspectiva mixta (cuantitativa y cualitativa).

Durante la experiencia fueron empleados métodos del nivel empírico, tales como: observaciones, el análisis de documentos, encuestas, entrevistas y otros, tanto en la fase de planificación para identificar las necesidades y precisar el problema científico, como en la fase de implementación y evaluación, para valorar la marcha del proceso. Además, se elaboró y aplicó un instrumento de diagnóstico inicial para conocer los datos generales de los participantes y su nivel de partida, con vista a adecuar el diseño curricular a sus necesidades, así como un cuestionario final para conocer los criterios de los participantes acerca del entrenamiento y su perfeccionamiento futuro.

Asimismo, durante la etapa de planificación fueron utilizados los métodos del nivel teórico: analítico-sintético, sistémico-estructural y modelación, entre otros, a partir de los cuales fue elaborada la primera versión de la propuesta, conformada por el programa, las actividades docentes, el sistema de trabajo independiente, el sistema de medios, entre otros elementos del diseño curricular del entrenamiento. De igual forma, los métodos del nivel matemático se usaron para sintetizar los datos en la evaluación de la propuesta.

Ahora bien, la recolección de los datos fundamentales de la experiencia fueron aportados a través de la valoración del desempeño de los personas entrenadas en los roles de autores y revisores de artículos, lo cual se explica en el apartado que sigue.

\subsection{La recolección de datos para evaluar el desempeño de las personas entrenadas}

Según se explicó en la introducción de este trabajo, la publicación de artículos en revistas, es un proceso lento y complejo, que requiere de una sistematización permanente de los interesados en publicar, por lo que es muy difícil que una acción de superación aislada pueda dar resultados inmediatos. 
En el caso que se presenta, como se verá más adelante, el entrenamiento fue concebido para desarrollarse en la modalidad semi-presencial, tres horas semanales durante cinco semanas; por ello, se conocía de antemano que no era posible esperar cambios profundos en el dominio de los contenidos por las personas entrenadas como resultado de la intervención mediante el entrenamiento. Precisamente, dadas las limitaciones para comparar los resultados antes y después de la implementación de la propuesta, es que el trabajo fue ubicado en la categoría de experiencia.

Sin embargo, independientemente de que no era plausible la comparación del estado final con el inicial, sí se pudieron recoger datos del desempeño de las personas entrenadas, a partir de la valoración de un grupo de indicadores que se midieron como parte del entrenamiento desde dos miradas: el desempeño de cada entrenado, tanto en el rol de autor, como en el rol de revisor; dichas valoraciones fueron realizadas mediante el análisis de los artículos entregados y las revisiones realizadas. Los datos recolectados permitieron valorar el comportamiento de cada uno de las personas entrenadas en los indicadores seleccionados y realizar el análisis de los datos de cada indicador desde una perspectiva integral. Además, se analizaron también los datos cualitativos recogidos en los instrumentos aplicados y en los criterios aportados durante la implementación.

Seguidamente se presentan los indicadores escogidos (identificados por las letras ordenadas alfabéticamente en el intervalo a-h), los cuales representan aspectos que, por lo general, las revistas científicas consideran entre sus estándares de calidad; se incluyen además las escalas ordinales empleadas para la medición, a partir de tres niveles de dominio: Bajo (1), Medio (2) y Alto (3).

Indicador a.- Ajuste al perfil y formato exigido por la revista.

- $\quad$ NIVEL ALTO (3): El artículo se ajusta al perfil de la revista seleccionada y cumple el formato exigido.

- NIVEL MEDIO (2): El artículo se ajusta en forma parcial al perfil y formato exigido por la revista seleccionada.

- $\quad$ NIVEL BAJO (1): El artículo no se ajusta al perfil y formato exigido por la revista seleccionada.

Indicador b.- Originalidad y actualidad del artículo.

- $\quad$ NIVEL ALTO (3): El artículo cumple satisfactoriamente los requisitos de originalidad y actualidad.

- $\quad$ NIVEL MEDIO (2): El artículo cumple de forma parcial los requisitos de originalidad y actualidad.

- $\quad$ NIVEL BAJO (1): El artículo no cumple los requisitos de originalidad y actualidad.

Indicador c.- Estructura del artículo.

- $\quad$ NIVEL ALTO (3): La estructura del artículo cumple de forma satisfactoria las exigencias de la revista seleccionada.

- $\quad$ NIVEL MEDIO (2): La estructura del artículo cumple en parte las exigencias de la revista seleccionada.

- NIVEL BAJO (1): La estructura del artículo no cumple de forma con las exigencias de la revista seleccionada.

Indicador d.- Sustento teórico del artículo.

- $\quad$ NIVEL ALTO (3): El artículo muestra el sustento teórico requerido.

- $\quad$ NIVEL MEDIO (2): El artículo muestra en parte el sustento teórico requerido.

- $\quad$ NIVEL BAJO (1): El artículo no muestra el sustento teórico requerido. 
Indicador e.- Sustento metodológico del artículo.

- $\quad$ NIVEL ALTO (3): El artículo presenta el sustento metodológico exigido por la revista.

- $\quad$ NIVEL MEDIO (2): El artículo presenta en parte el sustento metodológico exigido por la revista.

- $\quad$ NIVEL BAJO (1): El artículo no presenta el sustento metodológico exigido por la revista.

Indicador f.- Calidad de la redacción del artículo.

- NIVEL ALTO (3): El artículo manifiesta una excelente calidad en la redacción.

- NIVEL MEDIO (2): El artículo manifiesta por lo general una adecuada redacción, aunque en algunas ocasiones se aprecian limitaciones.

- NIVEL BAJO (1): El artículo manifiesta dificultades en la calidad de la redacción.

Indicador g.- Citas y referencias mostradas en el artículo.

- NIVEL ALTO (3): El artículo cumple satisfactoriamente las citas y referencias exigidas por la revista.

- $\quad$ NIVEL MEDIO (2): El artículo cumple en parte con las citas y referencias exigidas por la revista.

- NIVEL BAJO (1): El artículo no cumple con las citas y referencias exigidas por la revista.

Indicador h.- Tablas y gráficos presentados en el artículo.

- NIVEL ALTO (3): El artículo presenta las tablas y gráficos requeridos atendiendo a su tipología.

- $\quad$ NIVEL MEDIO (2): El artículo presenta en parte las tablas y gráficos requeridos atendiendo a su tipología.

- $\quad$ NIVEL BAJO (1): El artículo no presenta las tablas y gráficos requeridos atendiendo a su tipología.

\section{Concepción e implementación de la propuesta}

En este apartado se presenta una breve caracterización del programa de entrenamiento elaborado, así como una panorámica general de su implementación en la práctica.

\subsection{Caracterización del programa de entrenamiento}

El entrenamiento, según los documentos normativos del Ministerio de Educación Superior en Cuba, es la forma de organización de la educación posgraduada que posibilita la formación básica y especializada de los graduados universitarios, particularmente en la adquisición de habilidades y destrezas y en la asimilación e introducción de nuevos procedimientos y tecnologías con el propósito de complementar, actualizar, perfeccionar y consolidar conocimientos y habilidades prácticas (MES, 2009; MES, 2004).

En el caso que se presenta, la concepción del entrenamiento estuvo dirigida hacia líderes científicos e investigadores experimentados en el área de las Ciencias Pedagógicas, por lo que se exigía a los interesados como pre-requisitos de admisión: el dominio de habilidades informacionales básicas para el trabajo en la web, 
así como un informe escrito de resultados de investigación inéditos que fuera publicable en una revista científica. Los pre-requisitos mencionados anteriormente, sentaban las bases para que, durante el proceso del entrenamiento, cada entrenado pudiera elaborar una primera versión de artículo, (según las normas de la revista seleccionada), así como participar como revisor de los trabajos entregados por los demás participantes.

En general, se consideró la proyección de una matrícula para cada edición entre seis y ocho participantes, de modo tal que se le pudiera brindar la ayuda requerida de forma individualizada. En el cuadro que sigue se presenta una síntesis de los elementos fundamentales del programa de entrenamiento en su versión final.

Cuadro 1 - Síntesis de los elementos fundamentales del programa de entrenamiento en su versión final

Título: La publicación de artículos en revistas científicas.

Objetivo general:

Asesorar a líderes científicos e investigadores experimentados en el área de las Ciencias Pedagógicas en los contenidos fundamentales para desempeñarse como autores y revisores de artículos en revistas científicas de impacto internacional.

Objetivos específicos:

1.- Caracterizar artículos en revistas científicas de impacto internacional relacionados con los resultados que se pretenden publicar.

2.- Conocer el proceso para la publicación de artículos en revistas científicas de impacto internacional.

3.-Analizar revistas científicas de impacto internacional cuyos perfiles coincidan con los resultados que se pretenden publicar.

4.- Seleccionar una revista con posibilidades para el envío de un artículo derivado de los resultados de la investigación.

5.- Redactar el artículo siguiendo las normas de publicación de la revista seleccionada.

6.- Actuar como revisor de artículos científicos.

7.- Realizar la autovaloración de su trabajo en el entrenamiento en el rol de autor y en el rol de revisor.

Contenidos fundamentales:

El artículo científico. Las revistas científicas en línea. Recomendaciones generales para el proceso de elaboración de artículos para enviar a revistas científicas. La búsqueda y selección de revistas científicas en el área educativa. Las normas de publicación. Las formas de envío de manuscritos. El proceso editorial en las revistas científicas. La revisión de artículos en las revistas científicas.

Evaluación:

En el sistema de evaluación será considerado el trabajo realizado en las clases y el trabajo independiente y tendrá un peso fundamental la valoración del cumplimiento de las tareas del entrenado en los roles de autor y de revisor de artículos.

Fuente: Las autoras (2018).

\section{2.- Implementación del Entrenamiento}

Como se ha planteado, el entrenamiento se implementó en la modalidad semi-presencial, que presupone una carga docente mínima en las actividades presenciales y una carga más elevada dedicada al trabajo independiente de los participantes. Fue utilizada como forma de docencia la clase encuentro, con un tiempo de trabajo presencial de tres horas semanales, durante cinco semanas.

La matrícula inicial fue de ocho profesores, de ellos: cinco doctores, tres másteres, cuatro profesores titulares, tres auxiliares y un asistente. Con respecto a las funciones que desempeñan: tres eran jefes de proyectos de 
investigación, dos jefes de tarea, dos jefes de departamentos y un Vice-decano de investigación. La matrícula final fue de siete personas, ya que uno de los matriculados abandonó el entrenamiento.

Se diseñaron todas las guías para el trabajo independiente, las cuales contenían las tareas que las personas entrenadas debían desarrollar como parte de la preparación para los encuentros presenciales. A continuación se analizarán brevemente las actividades docentes desarrolladas.

En la Actividad docente 1 fueron analizados los documentos generales del entrenamiento (programa y esquema de contenidos), se aplicó el instrumento de diagnóstico y se discutieron las ideas de las personas entrenadas relacionadas con los conocimientos básicos de partida, así como los resultados inéditos que se pretendían publicar en las revistas de impacto internacional. Asimismo, fue orientada la guía para el trabajo independiente del siguiente encuentro, que contenía las tareas que aparecen en el cuadro que sigue.

\section{Cuadro 2 - Guía de trabajo del encuentro 2}

\section{GUÍA ENCUENTRO 2}

1.- Realice una búsqueda en la web de artículos contenidos en revistas, que estén relacionados con el tema del resultado que pretende publicar. Utilice como buscador el Google académico (http://www.scholar.google.com). Descargue al menos 3 artículos, uno de ellos escrito en idioma inglés y guárdelos convenientemente.

2.- Realice una búsqueda de artículos de revistas relacionados con su tema de investigación en la web utilizando la Base de Datos EBSCO. Descargue al menos 3 artículos y guárdelos convenientemente.

3a).- Elabore las fichas bibliográficas de los artículos descargados de la web.

b).- Utilizando las fichas de los artículos descargados de la web escriba el formato de presentación de los mismos como referencias bibliográficas según las normas APA.

Ambas tareas se deben entregar por escrito el día del encuentro.

4a).- Seleccione uno de los artículos descargados que muestre los resultados finales o parciales de una investigación. El artículo debe evidenciar de forma explícita el camino metodológico seguido por el autor para encontrar los resultados científicos.

b).- Analice la estructura, contenido y la metodología del artículo seleccionado siguiendo los pasos que se describen a continuación.

I.- Explicar la estructura que presenta el artículo.

II.- Describir brevemente el contenido de cada una de sus partes, destacando la metodología seguida para la obtención del resultado.

III.- Precisar la cantidad, actualidad y tipos de fuentes bibliográficas que aparecen registradas en el listado final del artículo utilizando la tabla que aparece seguidamente.

IV.- Argumentar cuáles de las fuentes citadas pudieran resultar de interés en su trabajo de investigación.

Fuente: Las autoras (2018).

En la Actividad docente 2 fueron analizadas las respuestas a las tareas orientadas, se destacó de manera especial, la presentación por parte de los estudiantes de los artículos seleccionados y se profundizó en la estructura del artículo científico y en los estándares de calidad que por lo general son exigidos por las revistas científicas (aspectos que posteriormente se emplearían como indicadores en la revisión de los artículos). En la explicación fueron utilizados como medios: la presentación de diapositivas, así como ejemplos de artículos y plantillas de revisión de revistas. Por último, fueron explicados los contenidos correspondientes al próximo encuentro y se orientaron las tareas correspondientes que se muestran en el siguiente cuadro. 
Cuadro 3 - Guía de trabajo del encuentro 3

\title{
GUÍA ENCUENTRO 3
}

1a).- Realice la búsqueda de al menos tres revistas de impacto internacional que en un primer acercamiento puedan resultar de interés para el posible envío de su artículo.

b) Descargue toda la información que se brinde en los sitios web de cada una de las revistas (presentación, perfil, cuerpo editorial, frecuencia de salida, indexaciones, normas para autores, plantillas de revisión de expertos, formas de envío, entre otros aspectos de interés).

c) Estudie detenidamente la información recopilada.

2.- Seleccione la revista que en una primera aproximación considere más conveniente para enviar su artículo. Argumente su selección.

3.- Realice la búsqueda de artículos publicados en la revista seleccionada que estén vinculados con su tema de investigación, que sirvan como modelo y que puedan ser utilizados como referencias bibliográficas en su artículo.

\section{Fuente: Las autoras (2018).}

En la Actividad docente 3 se analizó el cumplimiento de las tareas orientadas, se dedicó especial atención a los aspectos a considerar para la selección de revistas y se mostraron algunos ejemplos. Asimismo, se explicó que en la siguiente actividad debían entregar la primera versión del manuscrito con vistas al desarrollo del proceso de revisión, así como las normas para autores exigidas por la revista seleccionada y la plantilla de revisión si estuviese publicada.

La Actividad docente 4 se inició con el análisis del cumplimiento en la entrega del artículo y las normas de la revista para desarrollar el proceso de revisión. A continuación se explicó el proceso editorial en las revistas científicas, con énfasis en el método de revisión por expertos a ciegas y se mostraron ejemplos de evaluación de manuscritos, se destacó el uso de panel de revisión del Word y las plantillas de las revistas. Por último, fue explicado el proceso de revisión que debían seguir de los artículos que recibirían por el correo electrónico y se orientó la guía que contenía las tareas a realizar, las que se muestran en el cuadro que sigue.

\section{Cuadro 4 - Guía de trabajo del encuentro 5}

\author{
GUÍA ENCUENTRO 5 \\ 1.- Estudiar las normas de publicación y la plantilla de revisión de la revista seleccionada por el autor del \\ artículo que le corresponde evaluar. \\ 2.- Realizar la evaluación del artículo, utilizando el panel de revisión, destacando detalladamente los \\ comentarios y sugerencias al autor. \\ 3.- Presentar un informe de evaluación del artículo atendiendo a los aspectos siguientes: \\ a) Ajuste al perfil y formato de la revista; b) Originalidad y actualidad; c) Estructura; d) Sustento teórico; e) \\ Sustento metodológico; f) Calidad de la redacción; g) Citas y referencias; h) Tablas y gráficos.
}

Fuente: Las autoras (2018).

Con relación al desarrollo de la Actividad docente 5, cada entrenado presentó el informe del artículo que le correspondió revisar, sin hacer alusión al nombre del autor del artículo para mantener el anonimato, se mostraron las principales dificultades, así como las sugerencias para la mejora de estos; posteriormente a través del debate de las limitaciones detectadas, se pudieron establecer las regularidades fundamentales que mostraron los manuscritos entregados. Por último, se analizó de manera integral el entrenamiento desarrollado, 
se aplicó el instrumento de salida y se orientó el trabajo final del entrenamiento, el que sería enviado a los participantes por el correo electrónico.

Este trabajo final tenía como propósito que las personas entrenadas analizaran las revisiones finales de los artículos. Para ello, las evaluaciones de los profesores fueron ejecutadas en el propio documento del manuscrito revisado por las personas entrenadas en el rol de expertos, los cuales fueron compactados en una carpeta, para su envío como adjunto en un mensaje dirigido a todas las personas entrenadas, que se transcribe en el cuadro que se muestra a continuación.

\title{
Cuadro 5 - Mensaje final
}

\begin{abstract}
Estimados colegas:
Le enviamos en adjunto las revisiones finales de los artículos presentados por los participantes en el entrenamiento. Consideramos que sería muy conveniente que todos analizaran los señalamientos realizados por los profesores y los revisores-entrenados a los artículos entregados, por lo que esto puede representar en el mejoramiento del aprendizaje en los temas abordados. Por tal razón, se incluye esta actividad final de carácter no presencial que será incluida como parte de la evaluación final del entrenamiento.

TRABAJO FINAL DE CIERRE DEL ENTRENAMIENTO

1.- Exprese la autoevaluación de su actividad en el entrenamiento como autor de artículo científico.

Para ello seleccione la revisión del artículo elaborado por usted en la carpeta que se adjunta y analice las consideraciones de los revisores.

2.- Exprese la autoevaluación de su actividad en el entrenamiento como revisor de artículo científico.

Se recomienda para elaborar la tarea que seleccione el artículo revisado por usted en la carpeta que se adjunta y analice las consideraciones de los demás revisores.

3.- Haga una valoración de los resultados generales del entrenamiento en cuanto a la elaboración de artículos para revistas científicas, señalando las principales fortalezas y debilidades que se manifiestan en los artículos presentados por las personas entrenadas.

Para cumplimentar esta tarea recomendamos que estudie las revisiones de los artículos contenidos en la carpeta que se adjunta y valore críticamente los mismos.

Nota: la tarea final debe elaborarla en un documento word que debe ser enviado como adjunto al correo electrónico de las profesoras del entrenamiento.
\end{abstract}

Fuente: Las autoras (2018).

\section{Resultados y discusión}

En este apartado se analizarán los resultados obtenidos durante el entrenamiento a partir de la medición de los indicadores seleccionados a los que se hizo referencia en el epígrafe anterior. A continuación se presentarán los datos recolectados, para después realizar el análisis por personas entrenadas y por indicadores, se incluye además, la valoración cualitativa ofrecida por los participantes.

La tabla 1 muestra la evaluación de los indicadores (I) para cada una de las personas entrenadas (representados por números), desde la perspectiva de autor $(A)$ y como revisor $(R)$. 
Tabla 1 - Evaluación de indicadores por personas entrenadas como autor (A) y revisor(R)

\begin{tabular}{lllllllllllllll}
\hline $\mathrm{E}$ & $1 \mathrm{~A}$ & $1 \mathrm{R}$ & $2 \mathrm{~A}$ & $2 \mathrm{R}$ & $3 \mathrm{~A}$ & $3 \mathrm{R}$ & $4 \mathrm{~A}$ & $4 \mathrm{R}$ & $5 \mathrm{~A}$ & $5 \mathrm{R}$ & $6 \mathrm{~A}$ & $6 \mathrm{R}$ & $7 \mathrm{~A}$ & $7 \mathrm{R}$ \\
$\mathrm{l}$ & & & & & & & & & & & & & & \\
\hline $\mathrm{a}$ & 2 & 2 & 2 & 2 & 2 & 2 & 1 & 2 & 3 & 1 & 2 & 1 & 1 & 2 \\
$\mathrm{~b}$ & 3 & 3 & 2 & 3 & 3 & 2 & 2 & 2 & 3 & 2 & 2 & 2 & 2 & 3 \\
$\mathrm{c}$ & 3 & 2 & 1 & 1 & 1 & 3 & 1 & 1 & 2 & 2 & 1 & 1 & 1 & 2 \\
$\mathrm{~d}$ & 3 & 2 & 1 & 1 & 1 & 3 & 1 & 2 & 1 & 3 & 1 & 1 & 1 & 1 \\
$\mathrm{e}$ & 2 & 2 & 2 & 2 & 1 & 2 & 1 & 1 & 2 & 2 & 2 & 2 & 1 & 2 \\
$\mathrm{f}$ & 3 & 2 & 1 & 2 & 2 & 2 & 1 & 2 & 3 & 3 & 2 & 1 & 1 & 3 \\
$\mathrm{~g}$ & 2 & 3 & 1 & 1 & 1 & 2 & 1 & 1 & 1 & 3 & 1 & 1 & 1 & 2 \\
$\mathrm{~h}$ & 1 & 1 & 1 & 1 & 1 & 1 & 1 & 2 & 1 & 2 & 1 & 1 & 1 & 1 \\
\hline
\end{tabular}

Fuente: Las autoras (2018).

Los datos recolectados al finalizar el entrenamiento permitieron valorar el comportamiento de cada uno de las personas entrenadas en los indicadores seleccionados, así como la evaluación de cada indicador, desde una perspectiva integral. Seguidamente, se precisan las principales limitaciones presentadas.

\subsection{Análisis por personas entrenadas}

- Entrenado 1: las limitaciones fundamentales presentadas por este estudiante estuvieron referidas a la no utilización de tablas y gráficos para sintetizar la información. Por lo general, se aprecia correspondencia entre las evaluaciones obtenidas como autor y revisor.

- Entrenado 2: en este caso las insuficiencias, tanto en el rol de autor como de revisor se centraron en la estructura, el sustento teórico, en el trabajo con el empleo de citas y referencias de interés internacional, así como en lo referido al empleo de tablas y gráficos; además se manifestaron dificultades como autor en la calidad de la redacción.

- Entrenado 3: como autor, el estudiante manifestó limitaciones en la estructura del artículo y en los aspectos de orden metodológico, asimismo se apreciaron carencias en el marco teórico, ya que faltaron argumentos y referencias de interés en el contexto internacional y en el empleo de tablas y gráficos para sintetizar la información. Sin embargo, llama la atención el comportamiento seguido por el entrenado en el plano de revisor, pues pudo reconocer las principales limitaciones del manuscrito revisado.

- Entrenado 4: en el rol de autor manifiesta serias limitaciones en casi todos los aspectos evaluados, destacándose las dificultades en la terminología empleada no acorde al contexto internacional. En el rol de revisor, no pudo reconocer las limitaciones del manuscrito revisado en lo referido a la estructura y metodología, el empleo de citas y referencias, así como las tablas y gráficos.

- Entrenado 5: las limitaciones fundamentales de este participante como autor estuvieron referidas a las pobres argumentaciones teóricas desde la perspectiva internacional y el empleo de citas y referencias en revistas de la web, así como la no utilización de tablas y gráficos para sintetizar la información. En el plano de revisor, pudo reconocer las principales limitaciones del manuscrito revisado, excepto en lo referido al ajuste del tema del manuscrito a la revista seleccionada.

- Entrenado 6: como autor, las insuficiencias en este caso se centraron en la estructura, el sustento teórico, en el trabajo con el empleo de citas y referencias de interés internacional, así como en lo referido al empleo de tablas y gráficos. En el rol 
de revisor por lo general, se apreció un trabajo superficial en la mayoría de los indicadores seleccionados.

- Entrenado 7: manifiesta serias limitaciones como autor en casi todos los aspectos evaluados, se destaca por las pobres valoraciones críticas, la insuficiencia de las fuentes referenciadas y la falta de precisión en la esencia científica del trabajo. En el rol de revisor, no pudo reconocer las limitaciones del manuscrito revisado en lo referido al sustento teórico y al empleo de tablas y gráficos para sintetizar la información.

En general, se puede resumir que aún los docentes participantes en el entrenamiento presentan limitaciones en la calidad del artículo científico. Se considera importante valorar algunas cuestiones relativas al nivel de partida de los personas entrenadas, que afloraron en los datos obtenidos de la aplicación del instrumento inicial, así como en la discusión en la primera actividad docente, entre las que se acentúan: las limitaciones en el uso de las herramientas de la web, en especial, el trabajo con las bases de datos y revistas de impacto internacional, así como en la búsqueda y recuperación de información utilizando buscadores especializados, lo cual, a juicio de los autores de este artículo y profesores del entrenamiento, tuvo una influencia significativa en el aprendizaje de los contenidos por las personas entrenadas.

Asimismo, llama la atención también, como en los resultados de la encuesta inicial, casi todos las personas entrenadas evaluaron su preparación para la elaboración de artículos, en el nivel medio y sin embargo ya habían reconocido que casi nunca habían realizado consultas en artículos de revistas de impacto internacional; esto sugiere el desconocimiento de los participantes acerca de los estándares de calidad de los artículos publicados en esas revistas, así como la complejidad y rigurosidad que conlleva el proceso de elaboración del manuscrito. Las cuestiones señaladas pueden ser consecuencia de una cultura instalada durante muchos años que privilegió la publicación de ponencias en memorias de eventos, las que realmente no se correspondían con las exigencias actuales de un artículo científico.

Por otra parte, el comportamiento de la asistencia a las actividades docentes y el cumplimiento de las tareas de trabajo independiente estuvieron limitados durante el desarrollo del entrenamiento por diversas causas, que constituyeron barreras en la calidad del aprendizaje.

\subsection{Análisis por indicadores}

Para simplificar el tratamiento de los datos por indicadores se presenta la tabla 2, la cual muestra la evaluación relativa por indicadores (expresada en porcentaje) a partir de los datos obtenidos como autor y revisor en cada caso.

Tabla 2- Evaluaciones relativas por indicadores

\begin{tabular}{l|c|c|c}
\hline \multirow{2}{*}{\multicolumn{1}{c|}{ Indicadores }} & \multicolumn{3}{c}{ Evaluación de indicadores } \\
\cline { 2 - 4 } & Bajo(1) & Medio(2) & Alto(3) \\
\hline Indicador a & $28 \%$ & $64 \%$ & $7 \%$ \\
Indicador b & $0 \%$ & $57 \%$ & $43 \%$ \\
Indicador c & $57 \%$ & $28 \%$ & $14 \%$ \\
Indicador d & $64 \%$ & $14 \%$ & $21 \%$ \\
Indicador e & $28 \%$ & $75 \%$ & $0 \%$ \\
Indicador f & $28 \%$ & $43 \%$ & $28 \%$ \\
Indicador g & $64 \%$ & $21 \%$ & $14 \%$ \\
Indicador h & $93 \%$ & $7 \%$ & $0 \%$ \\
\hline
\end{tabular}

Fuente: Las autoras (2018). 
La comparación entre las evaluaciones de los indicadores de forma integral mostró entre los indicadores más afectados, el h relacionado con las tablas y gráficos; la mayoría de los autores no empleó estos recursos en su artículo o los empleó de forma incorrecta, mientras que los revisores tampoco se pronunciaron por su utilización. Asimismo, los indicadores d y g que representaban el sustento teórico y las citas y referencias también evidenciaron limitaciones, tanto en los roles de autores como en el de revisores, lo cual corrobora las carencias que en el desarrollo de habilidades informacionales aún están presentes en la muestra. Llama la atención el comportamiento del indicador c, relativo a la estructura del artículo, que también reportó índices bajos en las evaluaciones realizadas.

\subsection{Análisis cualitativo}

Por último, se hará referencia a los datos cualitativos obtenidos en la experiencia. No es posible en el marco de este artículo, por limitaciones de espacio, ofrecer toda la riqueza de los datos recogidos, los cuales aportaron los criterios y opiniones vertidas por las personas entrenadas a partir de los instrumentos aplicados y las autovaloraciones realizadas. Solo se mostrarán algunos ejemplos, que se presentan en el cuadro que sigue.

\section{Cuadro 6 - Ejemplos de datos cualitativos}

"El entrenamiento me sirvió de mucho ya que me dio la posibilidad de navegar por sitios en los que por la carga de trabajo no había tenido tiempo de hacerlo, me trazó metas que debía alcanzar para la entrega y preparación de las tareas orientadas".

"El aprendizaje logrado ha sido muy positivo, logré conocer cuestiones esenciales de los diferentes grupos a publicar, cómo entrar a las diferentes plataformas y cómo puedo hacer evaluaciones de los trabajos a partir de las normas de publicación de cada una de las revistas".

"Considero que el entrenamiento nos brindó herramientas de trabajo para poder evaluar artículos para publicar, además nos vemos reflejados en ellos y cuidamos mejor la forma de escribir los nuestros".

"Los intercambios fueron fructíferos en las acotaciones que nos hicieron las profesoras, y los demás integrantes, ya que lo que no se observaba en uno se reflejaban en otros y el taller final dio frutos de lo recibido durante los encuentros, elevando un poco más nuestra cultura informacional".

"Todas las actividades me aportaron a mi desarrollo profesional y a tomar conciencia de la necesidad de no cansarme y seguir enviando trabajos a diferentes revistas para su publicación".

"De las actividades realizadas me ha aportado más la revisión del artículo porque me permitió reflexionar en aspectos importantes que desde mi propio artículo no tuve en cuenta".

"Pienso que lo que atenta en el desarrollo del entrenamiento es la coincidencia de actividades atendiendo a las responsabilidades que debemos enfrentar".

"Considero que se debe sugerir a los directivos que podamos tener más tiempo para revisar revistas y artículos y profundizar en las cuestiones analizadas en el entrenamiento".

"Creo que tengo que profundizar más en mi auto-preparación en el estilo de redacción para que sea más comprensible por todos".

"Considero que debo organizarme y ponderar cuáles son las actividades que debo cumplir atendiendo a mi grado científico y docente, para lograr las publicaciones”.

"Coincido en los señalamientos planteados por los demás revisores, aunque debo ser más exigente a la hora de hacer este tipo de revisión si de verdad queremos ayudar a los autores". 
Como puede apreciarse, en los criterios mostrados, las personas entrenadas reconocen sus avances, así como sus propias limitaciones en los contenidos tratados, lo cual puede ser muy positivo para continuar su aprendizaje de forma independiente para elevar la cultura informacional, así como para cumplir sus funciones científicas y docentes con el uso de herramientas de trabajo actualizadas. Se advierte, además, una opinión favorable sobre la calidad de las actividades desarrolladas en el entrenamiento, por el aporte que brindaron al desarrollo profesional de los participantes.

\section{Conclusiones}

El análisis de los datos demostró las limitaciones que aún subsisten en los personas entrenadas con relación a la elaboración de artículos para publicar en las revistas científicas, sin embargo se reconoce la influencia que tuvo el entrenamiento en los discretos avances logrados en el dominio de los contenidos tratados y en especial en la toma de conciencia de los participantes acerca de la necesidad de elevar el rigor y la calidad en sus producciones científicas. El hecho de lograr que todas las personas entrenadas entregaran sus artículos, que participaran en el proceso de revisión de los mismos y que además pudieran estudiar y analizar los señalamientos realizados por los profesores y revisores-entrenados, ya se puede considerar como un logro del entrenamiento.

Es importante destacar, que el proceso de escritura requiere de una alta dosis de esfuerzo, dedicación y perseverancia por parte de los interesados en publicar en una revista científica de impacto internacional; esta capacidad no se puede alcanzar de inmediato a través de un curso o un entrenamiento, sino que es un proceso de adquisición sistemática de normas y prácticas inherentes al oficio de científico que depende en gran medida de la disposición del interesado en alcanzar la publicación.

En estos momentos, el programa de entrenamiento se continua implementando y se ha generalizado hacia otras facultades de la universidad, así como instituciones de la provincia. Además, el programa de superación profesional se brinda como parte de los servicios internacionales que ofrece la universidad a profesores de otros centros educacionales a través de cursos o pasantías y se trabaja en el montaje del entrenamiento en plataformas virtuales interactivas para ofrecer el servicio en la modalidad en línea.

\section{Referencias bibliográficas}

Antúnez, et al., (2012). Curso virtual de redacción científica e infotecnología sobre la plataforma MOODLE: resultados y experiencias en Píxel-Bit. Revista de Medios y Educación, 41, 173-183. Disponible en:

http://acdc.sav.us.es/pixelbit/images/stories/p41/13.pdf [Consultado el 3 de marzo de 20015]

Antúnez, G. (2006). Curso online Redacción Científica y las Tecnologías de la Información y las Comunicaciones. Revista Electrónica de Veterinaria REDVET, VII(8). Disponible en: http://www.veterinaria.org/revistas/redvet/n080806.html [Consultado el 10 de marzo de 2015]

Alfonso, J. E. (2004). Curso a distancia: Cómo preparar y publicar artículos científicos. Disponible en: http://files.sld.cu/redenfermeria/files/2011/05/tesis-final-1-03.pdf [Consultado el 15 de febrero de 2015]

Aramburuzabala, P., Reyes, R. \& I. Ángel, (2013). Modelos y tendencias de la formación docente universitaria. Revista Profesorado, 17 (3). Disponible en: http://www.ugr.es/local/recfpro/rev173COL9.pdf [Consultado el 20 de sept. de 2014]

Asencio, E. \& Ibarra, N. (2013). El empleo de revistas reconocidas de la web como fuentes de información en las tesis doctorales" en: Biblioteca digital "Félix Varela". Disponible en: http://fcp.uclv.cu [Consultado el 10 de febrero de 2014]

Cervantes, M. A., \& Martínez, R. (2014). Producción científica y redes de conocimiento: caso segunda generación del doctorado de ciencias administrativas. Raximhai, 10(5). Disponible en:

http://sistemanodalsinaloa.gob.mx/archivoscomprobatorios/ 10 articulosrevistasarbitraje/454.pdf [Consultado el 10 de mayo de 2015]

Colmenares, M. \& Piñero M. L. (2008). La investigación acción: una herramienta metodológica heurística para la comprensión y transformación de realidades y prácticas socio-educativas. Revista Laurus, 14 (27), 96-114. 
Cordero, G. et al., (2009). Publicación científica y evaluación docente en México. Tiempo de Educar, 10(19), 149-168. Disponible en: https://www.researchgate.net/profile/Jose Maria Garcia Garduno/publication/259198093 [Consultado el 15 de febrero de 2015]

Córdoba, G. S. (2005). Qué es una revista indexada. Girasol Digital, 8(26). Disponible en: http://www.vinv.ucr.ac.cr/girasolediciones/archivo/girasol26/indexada.htm [Consultado el 15 de sept de 2013]

Cruz, K. A. (2015). La formación inicial de investigadores. Raximhai, 11(4), 91-100. Disponible en: http://raximhai.com.mx/Portal/index.php [Consultado el 15 de febrero de 2015]

Day, R. (2005). ¿Cómo escribir y publicar artículos científicos? Publicaciones de la Organización Panamericana de la Salud. Tercera edición en español. Washington, Publicaciones Ciencia y Técnica 598.

Estrada, A. (2006). Curso Redacción científica y publicaciones académicas. Facultad de Letras y Ciencias Humanas, Madrid, Universidad Nacional Mayor de San Marcos. Disponible en: http://redaccioncientifica.blogspot.com/ [Consultado el 15 de febrero de 2015]

Espino, S. (2015). La enseñanza de estrategias de escritura y comunicación de textos científicos y académicos a estudiantes de posgrado. Revista Mexicana de Investigación Educativa, 20(66), 959-976. Disponible en: http://search.ebscohost.com/login. aspx?direct=true\&db=zbh\&AN=109100907\&site=ehost-live [Consultado el 20 de mayo de 2015]

Fonseca, C., Lías, Y. M. \& R. Gamboa, (2015) Autores e información del artículo. Revista Atlante: Cuadernos de Educación y Desarrollo. Disponible en:http://www.eumed.net/rev/atlante/2015/05/talleres.html [Consultado el 15 de mayo de 2015]

Gil, J. L., Morales, M. \& Basantes, J. L. (2015). Una aproximación a la calidad universitaria a partir de los procesos de evaluación y acreditación. Revista Universidad \& Sociedad, 7(1). Disponible en: http://rus.ucf.edu.cu [Consultado el 20 de mayo de 2015]

Gómez, A., Jiménez, S., \& Moreles, J. (2014). Publicar en revistas científicas, recomendaciones de investigadores de ciencias sociales y humanidades. Revista Mexicana de Investigación Educativa, 19(60). Disponible en:

http://www.scielo.org.mx/scielo.php?script=sci serial\&pid=1405-6666\&lng=es\&nrm=iso [Consultado el 15 de abril de 2015]

Gutiérrez, et al., (2009). Curso a distancia para la redacción de artículos científicos. Medisur, 7(2) disponible en:

http://www.medisur.sld.cu/index.php/medisur [Consultado el 15 de abril de 2015]

Hernández, A. (2010). Saberes, habilidades y estrategias utilizadas en la escritura de un texto científico: ensayo o artículo. Matices. Revista de posgrado, 5(13), 245-249. Disponible en:

http://revistas.unam.mx/index.php/matices/article/view/25800/24293 [Consultado el 10 de abril de 2015]

Mainegra, D., \& Miranda, J. (2012). Una propuesta para mejorar la comunicación de los resultados de la investigación educativa de la UCP "Rafael María de Mendive. Revista Mendive, 11(41).

MES (2004). Reglamento de la Educación de Posgrado de la República de Cuba. La Habana: Editado por el MES.

MES (2009). Modificaciones del Reglamento de la Educación de Posgrado de la República de Cuba. La Habana: Editado por el MES.

Miguel, S. (2011). Revistas y producción científica de América Latina y el Caribe: su visibilidad en SciELO, RedALyC y SCOPUS. Revista Interamericana de Bibliotecología, 34, (2), 187-199.

Ortega, M. F., Hernández, J. S., \& S. Tobón, (2015). Impacto de la cartografía conceptual como estrategia de gestión del conocimiento. Raximhai, 11(4), 171-180. Disponible en: http://raximhai.com.mx/Portal/index.php [Consultado el 15 de febrero de 2015]

Sabaj, O. (2009). Descubriendo algunos problemas en la redacción de Artículos de Investigación Científica de alumnos de postgrado. Revista Signos, 42(69), 107-127. Disponible en: http://www.scielo.cl/scielo.php?pid=S071809342009000100006\&script=sci arttext [Consultado el 10 de junio de 2012]

Sampiere, R. H., Collado, C. F., \& Lucio, P. B. (2006). Metodología de la investigación. Cuarta edición. México, M. G.-H. Interamericana (Ed.).

Sánchez, B. (2006). Ciencia, Investigación y Cultura en la biblioteca universitaria actual. Conferencia magistral presentada en la Academia de Ciencias de Cuba. La Habana. Disponible en: http://decs.bvs.br/E/homepagee.htm [Consultado el 20 de mayode2012]

Santovenia, J. R., \& Linares, M. P. (2011). Proponen curso de redacción de documentos científicos desde la perspectiva de las Ciencias de la información. Ciencias de la Información, 42(2), 71-73. Disponible en: http://www.idict.cu [Consultado el 15 de mayo de2013]

Suárez, C., Escalona, E. \& Boza, Y. (2012) Algunas consideraciones sobre el proceso de autoevaluación de la actividad científica educacional en las Universidades de Ciencias Pedagógicas. Revista Congreso Universidad, I (2). Disponible en:http://200.14.5.210/revista/index.php/congresouniversidad/article/viewFile/85/ [Consultado el 10 de junio de 2012] 
Valera, O. (2005) La información científica en la investigación educativa" en: Metodología de la investigación educacional. Desafíos y polémicas, 68-85, La Habana: Editorial Pueblo y Educación.

Villagrán, A. (2009). Algunas claves para escribir correctamente un artículo científico. Revista chilena de Pediatría, 80(1), 7078. Disponible en: http://www. scielo.cl/scielo.php?pid=S0370-1062009000100010\&script=sci arttext [Consultado el $15 \mathrm{de}$ febrero de 2015]

Villamón, M. et al., (2005). Análisis de la visibilidad de las revistas científico-técnicas españolas de ciencias de la actividad física y el deporte. Revista de Psicología del Deporte, 14(2), 253-267.

Zapata, C. M., \& Velásquez, J. D. (2008). Algunas pautas para la escritura de artículos científicos. Ingeniare. Revista chilena de ingeniería, 16(1), 128-137. Doi: http://dx.doi.org/10.4067/S0718-33052008000100002

\title{
Datos de las autoras
}

\author{
Esperanza Asencio Cabot
}

Doctora en Ciencias Pedagógicas. Profesora Titular Consultante del Departamento de Pedagogía- Psicología de la Facultad de Educación Infantil de la Universidad Central "Marta Abreu" de Las Villas. Cuba. Investiga en el área de la Educación científica y la Gestión de la información en las Ciencias Pedagógicas. Ha publicado varios libros y artículos en revistas educativas de impacto internacional, y ha trabajado como revisora de revistas educativas extranjeras de perfil educativo. Se desempeña actualmente como Editora científica de la Revista Varela y dirige un proyecto de investigación para promover la actualización en la Gestión de la Información Científico-Técnica de profesores y estudiantes de las Facultades de Educación de la Universidad Central "Marta Abreu" de Las Villas, así como de profesores y bibliotecarios del Ministerio de Educación en la provincia de Villa Clara.

easencio@uclv.cu

\section{Nilda Ibarra López}

Licenciada en Letras. Máster en Historia y Cultura de Cuba. Profesora auxiliar. Jefe de Sección de la Dirección de Documentación e Información Científico Técnica de la Universidad Central "Marta Abreu" de Las Villas. Cuba. Editora jefe de la Revista Varela. Investiga en el área de la Literatura y la Gestión de la información en las Ciencias Pedagógicas. Ha publicado artículos en revistas educativas de impacto internacional y ha trabajado como revisora de revistas educativas extranjeras de perfil educativo. Segunda Jefe de proyecto de investigación para promover la actualización en la Gestión de la Información Científico-Técnica de profesores y estudiantes de las Facultades de Educación de la Universidad Central "Marta Abreu" de Las Villas, así como de profesores y bibliotecarios del Ministerio de Educación en la provincia de Villa Clara.

\section{nibarra@uclv.cu}

Recibido - Received: 2017-07-16

Aceptado - Accepted: 2018-01-17

\section{(cc) EY}

This work is licensed under a Creative Commons Attribution 4.0

United States License.

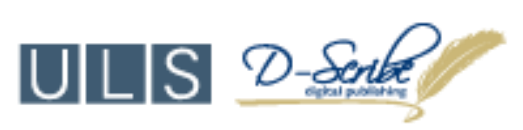

This journal is published by the University Library System of the University of Pittsburgh as part of its D-Scribe Digital Publishing Program and is cosponsored by the University of Pittsburgh Press. 